\title{
Computational Modeling Evidence of a Nonthermal Electromagnetic Interaction Mechanism With Living Cells: Microwave Nonlinearity in the Cellular Sodium Ion Channel
}

\author{
Nikolay S. Stoykov, Member, IEEE, Joseph W. Jerome, Lauren C. Pierce, and Allen Taflove, Fellow, IEEE
}

\begin{abstract}
A computational hydrodynamics model consisting of a system of four coupled time-domain partial differential equations is applied to study the response of the cellular sodium ion channel to a microwave electric-field excitation. The model employs a dynamic conservation law formulation, which has not been previously applied to this problem. Results indicate that the cellular sodium ion channel exhibits an electrical nonlinearity at microwave frequencies, which generates an intermodulation spectrum when excited by an amplitude-modulated electric field. Intermodulation products having frequencies down to $50 \mathrm{MHz}$, and very likely well below $50 \mathrm{MHz}$, appear possible. This is a new nonthermal microwave interaction mechanism with living tissues that, if observable below $0.1 \mathrm{MHz}$, could enable the stimulation of excitable biological tissues, and thereby have significant implications for human health and safety.
\end{abstract}

Index Terms-Computational modeling, living cells, microwaves, nonlinearity, nonthermal electromagnetic interactions.

\section{INTRODUCTION}

$\mathbf{T}$ HERE EXISTS a substantial literature regarding the biological effects of electromagnetic fields from ac power frequencies to microwaves [1]-[5]. However, in this body of research, relatively few studies have attempted to investigate potential interaction mechanisms at the level of individual cells.

This paper reports on the results of a detailed computational study of the response of the cellular sodium ion channel to an amplitude-modulated microwave electric-field excitation. We have extended and applied a hydrodynamic model that integrates a system of four coupled partial differential equations which includes the Poisson-Nernst-Planck (PNP) equations [6] as a limiting case. The simulation code for our model is adapted from that was created by C.-W. Shu, Brown University, Provi-

Manuscript received July 8, 2003; revised February 13, 2004. This work was supported in part by the National Science Foundation under Grant DMS-0311263.

N. S. Stoykov is with the Rehabilitation Institute of Chicago, Chicago, IL 60611 USA and also with the Department of Physical Medicine and Rehabilitation, Northwestern University, Chicago, IL 60611 USA (e-mail: n-stoykov@northwestern.edu).

J. W. Jerome is with the Department of Mathematics, Northwestern University, Evanston, IL 60208 USA.

L. C. Pierce is with the Weinberg College of Arts and Sciences, Northwestern University, Evanston, IL 60208 USA.

A. Taflove is with the Department of Electrical and Computer Engineering,

Northwestern University, Evanston, IL 60208 USA.

Digital Object Identifier 10.1109/TMTT.2004.831924 dence, RI, and is used in this paper with permission. A detailed description of the algorithm is provided in [7] and [8]; salient features are summarized in Section VI. We believe this to be the initial application of such modeling technology to the electromagnetic bioeffects problem.

Our computational model is based upon the dynamic conservation law formulation. In turn, the dynamic conservation law formulation is a natural extension of the steady-state conservation law model of [9]. The spatial dimensions of our model are in the order of nanometers in length and tenths of nanometers in diameter. The temporal events analyzed occupy time scales ranging from subpicoseconds to tens of nanoseconds, much less than the duration of gating pulses, which are typically in the millisecond range. We are thus examining the open channel.

Our model indicates that the cellular sodium ion channel exhibits an electrical nonlinearity at microwave frequencies, which generates an intermodulation spectrum when excited by an amplitude-modulated electric field. Intermodulation products having frequencies down to $50 \mathrm{MHz}$, and very likely well below $50 \mathrm{MHz}$, appear possible. This is a new nonthermal microwave interaction mechanism with living tissues that, if observable below $0.1 \mathrm{MHz}$, could enable the stimulation of excitable biological tissues [10] and thereby have significant implications for human health and safety.

\section{OVERVIEW OF THE STRUCTURE AND FUnCTION OF ION CHANNELS}

Sodium channels exist in axons and neuron cell bodies, in striated muscles and cardiac muscles, as well as in many endocrine glands [11]. Unlike potassium channels, they are very similar in function, but their kinetics differ. In axons and muscle fibers, fast sodium channels generate action potentials, which are the foundation of any sensory-motor performance and higher cognitive functions. Slow sodium channels, most clearly observed in the soma of cells in the hippocampus, neostriatum, thalamus, and other parts of the brain, regulate the excitability of cells by generating prolonged sub-threshold changes in the membrane potential [12]. Without actually initiating the action potential, they condition the cell and, thus, modulate the delicate mechanisms of activation of neuronal circuits. For example, slightly depolarized thalamic neurons oscillate at the frequency of approximately $10 \mathrm{~Hz}$, whereas in the hyperpolarized state, they are likely to oscillate at approximately $6 \mathrm{~Hz}$ [13]. 
The slow sodium channels give rise to the so-called noninactivating or persistent conductance [12]. It is conceivable that this prolonged conductance of sodium ions may expose the cell to ambient electric fields of frequencies below the gating frequency of the fast sodium channels. Such fields may, therefore, disrupt the function of neuronal circuits.

Ambient low-frequency electric fields disrupt the function of neuronal circuits because they can elicit an action potential. The new perspective we obtain in the context of the sodium channels is that these low-frequency fields may arise from a possible nonlinear response of the channel to microwave fields. Such nonlinear response may be expected to occur at some frequencies, not necessarily in the microwave region, if the ion flow through the channel is considered an electro-hydrodynamical process.

From this point-of-view, we expect a similar nonlinear response in any kind of ion channels. For example, resting channels may provide a high degree of coupling of an ambient electric field with the cell because they are open when the cell is at rest and, therefore, would not obstruct the flow of low-frequency intermodulation currents. In nerve cells, the resting channels are permeable to potassium, sodium, and chloride ions [14].

We believe that the ion flow through the channel can reasonably be considered an electro-hydrodynamical process because the ion channels themselves are thought to be water-filled pores [15]. Ions pass through the pores at the rate of $10^{8} / \mathrm{s}$ [16]. This model was not fully accepted until at least the late 1970s. Up to that time, an alternative hypothesis under investigation was that the ion channels are structures for active transport of ions [15].

Ion channels are formed by proteins spanning the cell membrane. Genetic research has shown that voltage-gated sodium, potassium, and calcium channels have evolved from a common gene and have the same overall structure [16], [17]. This similarity justifies to some extent generalizing results obtained for one type of channels to other types.

Experiments using high-resolution X-ray crystallography have revealed the three-dimensional (3-D) geometrical structure of a potassium channel [18]. The pore was found to be constructed of an "inverted teepee." The selectivity filter is situated at the wide end of the pore and is about 1.2-nm long, thus permitting two potassium ions to pass through it in close proximity one after the other. The repulsive force between the ions increases their speed in the filter. The width of the filter allows only a single potassium ion to pass by a given point along the filter at a time. This confirms a long-standing hypothesis that the ions are dehydrated before they pass through the filter [15]. A large water-filled cavity in the center of the pore reduces the electrostatic barrier in the middle of the lipid bilayer of the membrane.

Extrapolated to a sodium ion channel, these results justify an initial one-dimensional (1-D) approach to modeling the ion channel. However, such an approach neglects the fine structure of the water cavity and the overall conical shape of the channel. The electro-hydrodynamical concept excludes the process of dehydrating of the ions. If considered in a more general paradigm, these details might reveal some additional nonlinear effects.

\section{System of Coupled PARTIAL DifFEREnTIAL EQuations}

Our model of the response of the cellular sodium ion channel to an amplitude-modulated microwave electric field involves integrating in time $(t)$ a hydrodynamic model consisting of a system of four coupled time-domain partial differential equations that enforce conservation of particles, conservation of momentum, conservation of energy, and Poisson's equation (see [9, Appendix]). In this model, a 1-D space having the positional coordinate $x$ is considered. The system of equations is as follows.

\section{A. Conservation of Particles}

$$
\frac{\partial n}{\partial t}+\frac{\partial}{\partial x}(n v)=0
$$

where $n$ and $v$ are, respectively, the ion concentration and ion translational velocity in the channel's pore.

\section{B. Conservation of Momentum}

$$
\frac{\partial p}{\partial t}+\frac{\partial}{\partial x}(p v)+\frac{\partial}{\partial x}\left(n k_{B} T\right)=e n E-\frac{p}{\tau_{p}}
$$

where $p=m n v$ is the ion momentum density in the channel's pore, $m$ is the ion mass, $k_{B}$ is Boltzmann's constant, $T$ is the ion temperature in the channel's pore, $e$ is the electron charge, $E=-(\partial \Phi / \partial x)+E_{\text {ext }}$ is the total electric field, $\Phi$ is the electric potential, $E_{\text {ext }}$ is an externally applied electric field of arbitrary time waveform and intensity, and $\tau_{p}$ is the momentum relaxation time of the ions in the channel's pore.

\section{Conservation of Energy}

$$
\begin{aligned}
& \frac{\partial w}{\partial t}+\frac{\partial}{\partial x}(v w)+\frac{\partial}{\partial x}\left(n v k_{B} T\right) \\
& =e n v E-\frac{w}{\tau_{w}}+\frac{3 n k_{B} T_{0}}{2 \tau_{w}}+\frac{\partial}{\partial x}\left(\kappa n \frac{\partial T}{\partial x}\right)
\end{aligned}
$$

where $w=(3 / 2) n k_{B} T+(1 / 2) n m v^{2}$ is the ion energy density, $T_{0}$ is the temperature of the channel's protein and lipid membrane, $\tau_{w}$ is the energy relaxation time of ions in the channel's pore, $\kappa=\left(3 \tilde{m}_{0} k_{B}^{2} T_{0} / 2 e\right)$ is the thermo-conductivity coefficient of the ions, and $\tilde{m}_{0}$ is the ion mobility in the channel's pore when the electric field and other driving forces are small.

\section{Poisson's Equation}

$$
-\varepsilon \frac{\partial^{2} \Phi}{\partial x^{2}}=e\left(n+n_{D}\right)
$$

where $\varepsilon$ is the dielectric permittivity of the channel's pore, and $n_{D}$ is the distribution of permanent charge on the channel protein.

Equation (1) ensures conservation of the number of particles. Here, the concentration of particles changes with time solely as the result of drift (flow). In (2) and (3), collisions are approximated as relaxations to values of the equilibrium state. In each of these equations, the second term on the left-hand side is due to drift, and the third term is due to the pressure gradient, i.e., 
the mechanical force, which contributed from ion-ion interactions and ion thermal motion.

On the right-hand sides of (2) and (3), the first term is due to the electrical force. This arises from: 1) the charge applied to the baths that sustains the externally applied transmembrane potential; 2) the permanent charge on the channel protein; 3) the mobile charge (ions) in the channel's pore; 4) the induced (i.e., polarization) charge of the several dielectrics; and 5) the externally applied microwave field. The second term on the right-hand sides of (2) and (3) is due to the frictional force arising from ion-channel and ion-water interactions. Finally, the last term on the right-hand side of (3) is due to the heat flux into the system.

\section{BOUNDARY CONDITIONS}

We assume that the ion channel is positioned symmetrically between $x=-(L / 2)$ and $x=(L / 2)$, where $L$ denotes the channel length. At $x=-(L / 2)$, the potential is defined by the built-in potential and the bias across the channel

$$
\Phi\left(-\frac{L}{2}\right)=V_{\text {bias }}+\frac{k_{B} T}{e} \ln \left[\frac{n_{i}}{n\left(-\frac{L}{2}\right)}\right]
$$

where $n_{i}$ is the intrinsic ion concentration and $V_{\text {bias }}$ is a userdefined static potential. At $x=(L / 2), \Phi$ is defined exclusively by the built-in potential

$$
\Phi\left(\frac{L}{2}\right)=\frac{k_{B} T}{e} \ln \left[\frac{n_{i}}{n\left(\frac{L}{2}\right)}\right] .
$$

We note that, because of the way that the channel protein folds, there is a normally directed electric field within the lumen, resulting in a periodic potential along its length. However, we do not incorporate this periodicity in the boundary conditions in this model.

Consider next the boundary conditions on the carrier concentrations. These are given at $x= \pm(L / 2)$ by the values of the ion density

$$
n\left( \pm \frac{L}{2}\right)=n_{D}\left( \pm \frac{L}{2}\right) .
$$

Finally, the boundary condition temperatures are given by

$$
T\left(-\frac{L}{2}\right)=T_{0} \quad T\left(\frac{L}{2}\right)=T_{0}
$$

where $T_{0}$ is the ambient temperature. There are no boundary conditions directly specified for the velocity in the incompletely parabolic system of (1)-(3).

\section{PARAMETERS}

Following [9], we employ the Baccarani-Wordeman empirical models [19] for the relaxation parameters

$$
\begin{aligned}
\tau_{p} & =\frac{m \tilde{m}_{0} T_{0}}{e T} \\
\tau_{w} & =\frac{3 \tilde{m}_{0} k_{B} T T_{0}}{2 e v_{s}^{2}\left(T+T_{0}\right)}+\frac{\tau_{p}}{2}
\end{aligned}
$$

TABLE I

PARAMETERS USED IN THE MODEL

\begin{tabular}{ll}
\hline Parameter & Value \\
\hline$\varepsilon$ & $80 \varepsilon_{0}$ \\
$n_{D}$ & $10 \mathrm{mmol} \mathrm{l}^{-1}=6.02 \mathrm{~nm}^{-3}$ \\
$n_{i}$ & $0.1 \mathrm{~mol} \mathrm{l}^{-1}=0.0602 \mathrm{~nm}^{-3}$ \\
$v_{s}$ & $0.01 \mathrm{~nm} \mathrm{ps}^{-1}$ \\
$V_{\text {bias }}$ & $0.2 \mathrm{~V}$ \\
$m$ & 23 a.m.u. (mass of $\mathrm{Na}^{+}$) \\
$T_{0}$ & $300^{\circ} \mathrm{K}$ \\
$\tilde{m}_{0}$ & $0.04 \mathrm{~nm}^{2} \mathrm{ps}^{-1} \mathrm{~V}^{-1}$ \\
$L$ & $2.5 \mathrm{~nm}$ \\
\hline
\end{tabular}

where $v_{s}$ is the saturation velocity of ions in the channel's pore. The following parameters assumed for our model are summarized in Table I. These parameters were also used in [9] and are thought to be representative of the cellular ion channel. The choice of these parameters is nontrivial, clearly impacting the results. Of these parameters, most are well understood. There are two, however, that are less clear: the saturation velocity $v_{s}$ and mobility $\tilde{m}_{0}$. Note that, as discussed in [9], the current choice of $v_{s}$ is consistent with the convergence of the hydrodynamics model with decreasing relaxation time to the pnp model. The key assumption in selecting $\tilde{m}_{0}$ is that the diffusion in the pore is the same as that in a free solution [9].

\section{AlgORITHM}

The hydrodynamic model summarized above can generate a wide variety of nonlinear fluid behavior including the formation of propagating shock waves and other disturbances-indeed, much of the behavior of fluids that we observe every day as weather, water flows in our sinks and drains, and even waves at the beach. Simple discretization does not always work well for such systems, and can even lead to completely erroneous modeling results. Sophisticated numerical techniques must be applied to properly account for the possibility of shock production. For this reason, we employ the previously developed, essentially nonoscillatory (ENO) shock-capturing scheme described in detail in [7] and [8], and previously applied to model the open ion channel (unexcited by microwaves) in [9]. Several key aspects of the ENO algorithm and our adaptation of it for this study are now reviewed. (For the reader interested in a detailed mathematical description of the algorithm, [8] provides a readable and extensive discussion.)

First, and most generally, the ENO algorithm permits a flexible specification of its order of numerical accuracy. For this study, we select a third-order accurate numerical implementation in both space and time for the conservation law system, and use standard second-order differencing for Poisson's equation. For the conservation system, spatial differencing adaptively employs stencil subsets of a uniform grid that are selected to minimize oscillations, as discussed below. Time stepping is via an explicit third-order Runge-Kutta scheme, which permits variable time-step size. Before the algorithm can proceed to the next time step, two quantities must be computed: the Courant-Friedrichs-Levy (CFL) number (the numerical stability index that governs the length of the time step) and the 
numerical flux. Given the 2.5-nm-length $L$ of the ion channel, a subpicosecond time step is required both to accurately model ion flow and to ensure numerical stability. That is, velocities of ion transport integrated over subpicosecond time intervals yield translational distances within the channel that are relatively small compared with $L$ so that the results of the numerical integration are accurate.

Second, with our model written as a perturbed first-order hyperbolic system, the Jacobian matrix can be computed analytically together with its eigenvalues and eigenvectors. In fact, the eigenvalues are given by $v-c, c$, and $v+c$ for sound speed $c^{2}=\left(5 k_{B} T / 3 m\right)$. The eigenvalue with the largest modulus $\lambda$ determines the time step $(\Delta t / \Delta x) \leq(1 / \lambda)$. The eigenvectors permit a field-by-field decomposition so that, ultimately, the flux can be defined for single scalar equations and propagate to the system via similarity transformations. In this diagonalization, the boundary conditions for the numerical method are characterized as inflow boundary conditions, which are standard for hyperbolic problems. The algorithm is overdetermined (in the subsonic case) by requiring the velocity gradient to vanish, but this is known to converge to analytical compatibility as $\Delta x \rightarrow$ 0 .

Third, and most specifically, the ENO scheme is targeted at the numerical solution of scalar equations of the form

$$
\frac{\partial u}{\partial t}+\frac{\partial[f(u)]}{\partial x}=0
$$

which can develop shocks and/or steep gradients. The ingenuity of the ENO scheme lies in the numerical representation

$$
\frac{\partial[f(u)]}{\partial x}=\frac{\left(\hat{f}_{i+\frac{1}{2}}-\hat{f}_{i-\frac{1}{2}}\right)}{\Delta x}
$$

where $\hat{f}_{i-(1 / 2)}$ is the numerical flux flowing into a cell situated halfway between the grid points. It was shown in [7] that this value could be computed as the analytical derivative of a certain Newton interpolation polynomial constructed via finite differences on adaptive stencils, which minimize oscillations. The degree of the Newton polynomial is related to the order of the method in regions of smoothness of the solution, and is of degree 4 in our case. An additional comment relates to the decomposition of the flux, prior to approximation, into a part $f^{+}$ with nonnegative signal speed, and a part $f^{-}$with nonpositive signal speed. This is called the local Lax-Friedrichs flux splitting, and defines the upwinding. The interpolation polynomials are actually constructed for each flux component.

When the ENO algorithm is applied to perturbed equations (forcing terms added), the forcing terms are treated explicitly. This is the case even for the term involved in the energy equation where the heat-flux divergence is discretized by a classical second-order approximation. The model used here is called an incompletely parabolic system, defined by the hyperbolic subsystem for the concentration and momentum densities, and the parabolic energy equation for the carrier temperature. This affects the consistency of the boundary conditions.
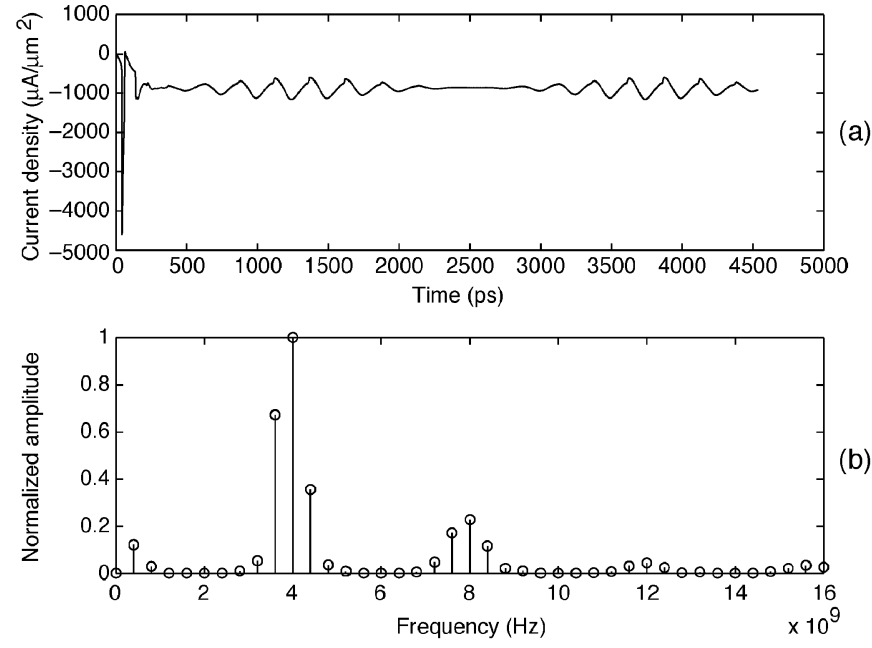

Fig. 1. (a) Computed time waveform of the sodium ion current density for a $20-\mathrm{V} / \mathrm{cm} 4-\mathrm{GHz}$ microwave excitation amplitude modulated at $100 \%$ by a 400-MHz sinusoid. (b) Corresponding Fourier spectrum with the dc component suppressed.

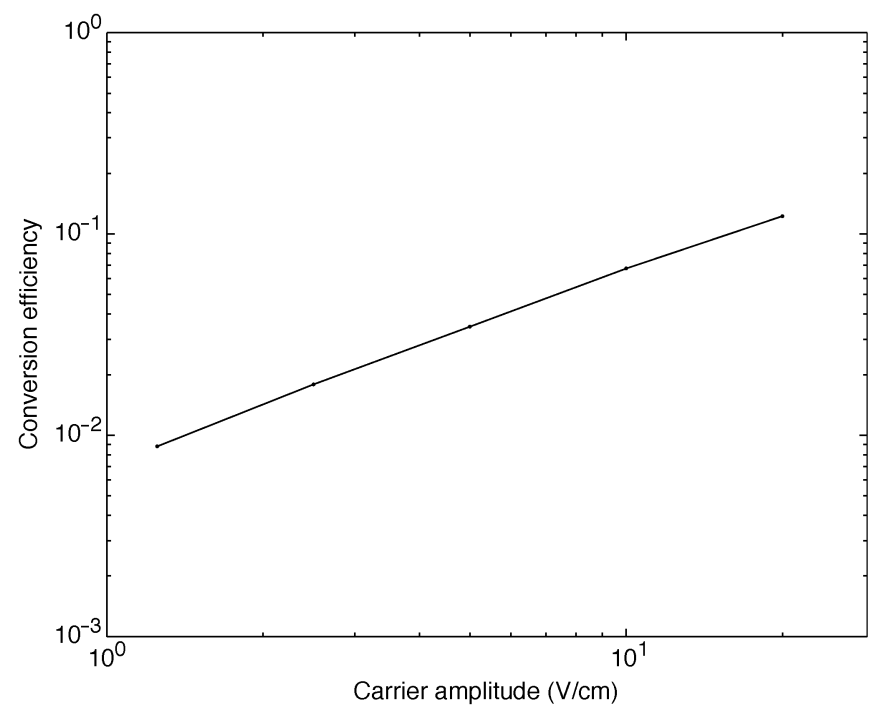

Fig. 2. Computed LFCE as a function of the 4-GHz carrier amplitude. The amplitude modulation by a $400-\mathrm{MHz}$ sinusoid is fixed at $100 \%$. Characteristic of nonlinear systems, the conversion efficiency diminishes exponentially as the carrier amplitude is reduced. With the carrier reduced to $1.25 \mathrm{~V} / \mathrm{cm}$, the LFCE drops to approximately $0.8 \%$.

\section{RESULTS}

Fig. 1(a) graphs the temporal waveform of the calculated sodium ion current density in the channel for a $20-\mathrm{V} / \mathrm{cm} 4-\mathrm{GHz}$ microwave electric-field excitation amplitude modulated at $100 \%$ by a $400-\mathrm{MHz}$ sinusoid. Fig. 1 (b) is the corresponding Fourier spectrum of this current density with the dc spectral component suppressed. This spectrum is normalized to the peak component at the 4-GHz carrier frequency. We observe intermodulation products clustered about the fundamental frequency and its harmonics. Such an intermodulation spectrum is characteristic of the excitation of a nonlinear system by multiple-frequency sinusoids. Of particular interest is the low-frequency product at the $400-\mathrm{MHz}$ modulation frequency. This product has an intensity that is approximately $12 \%$ of the 


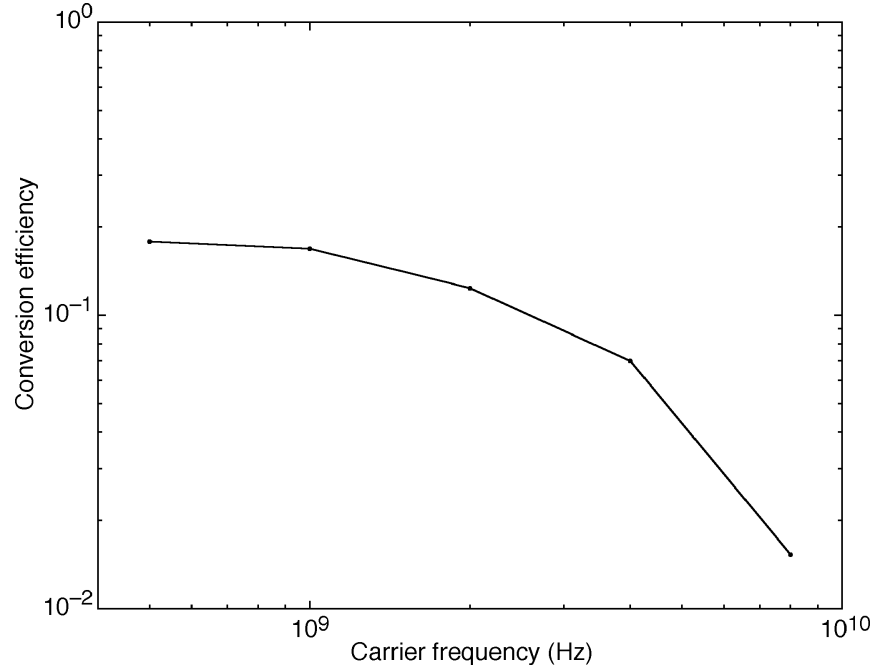

Fig. 3. Computed LFCE as a function of the microwave carrier frequency from $500 \mathrm{MHz}$ to $8 \mathrm{GHz}$, maintaining a $50-\mathrm{MHz}$ amplitude modulation at $100 \%$ and a carrier amplitude of $10 \mathrm{~V} / \mathrm{cm}$. The conversion efficiency is approximately constant for carrier frequencies below $1 \mathrm{GHz}$, but drops off exponentially for carrier frequencies above $5 \mathrm{GHz}$.

carrier. We define this ratio as the low-frequency conversion efficiency (LFCE).

Fig. 2 graphs the LFCE observed for the case of Fig. 1 as a function of the 4-GHz carrier amplitude. Characteristic of nonlinear systems, the LFCE diminishes exponentially as the carrier amplitude is reduced. With the carrier reduced to $1.25 \mathrm{~V} / \mathrm{cm}$, the LFCE drops to approximately $0.8 \%$.

Fig. 3 graphs the LFCE as a function of the carrier frequency from $500 \mathrm{MHz}$ to $8 \mathrm{GHz}$, maintaining a $50-\mathrm{MHz}$ amplitude modulation at $100 \%$ and a carrier amplitude of $10 \mathrm{~V} / \mathrm{cm}$. We see that the conversion efficiency is approximately constant for carrier frequencies below $1 \mathrm{GHz}$, but drops off exponentially for carrier frequencies above $5 \mathrm{GHz}$.

Fig. 4 graphs the LFCE as a function of the modulating frequency between $50-800 \mathrm{MHz}$, maintaining $100 \%$ amplitude modulation of a $20-\mathrm{V} / \mathrm{cm} 4-\mathrm{GHz}$ carrier. We see that the LFCE is approximately constant over the entire range of modulating frequencies.

\section{DISCUSSION}

Our computational modeling results imply that an amplitudemodulated microwave electric field can induce low-frequency ion currents in the cellular sodium channel due to a nonlinearity inherent in the ion-flow process. As discussed in Section II, the sodium channel influences the subtle operating mechanisms of neuronal circuits. Furthermore, because "there should be much mechanistic similarity among the channels" [17], our results should be generalizable to potassium and calcium channels.

We note that the subpicosecond time step used in our algorithm prevents integration to the time scales needed to directly calculate low-frequency mixing products below approximately $50 \mathrm{MHz}$. However, Fig. 4 indicates that reducing the modulation frequency results in an undiminished LFCE down to $50 \mathrm{MHz}$, and very likely well below $50 \mathrm{MHz}$. Given that "below

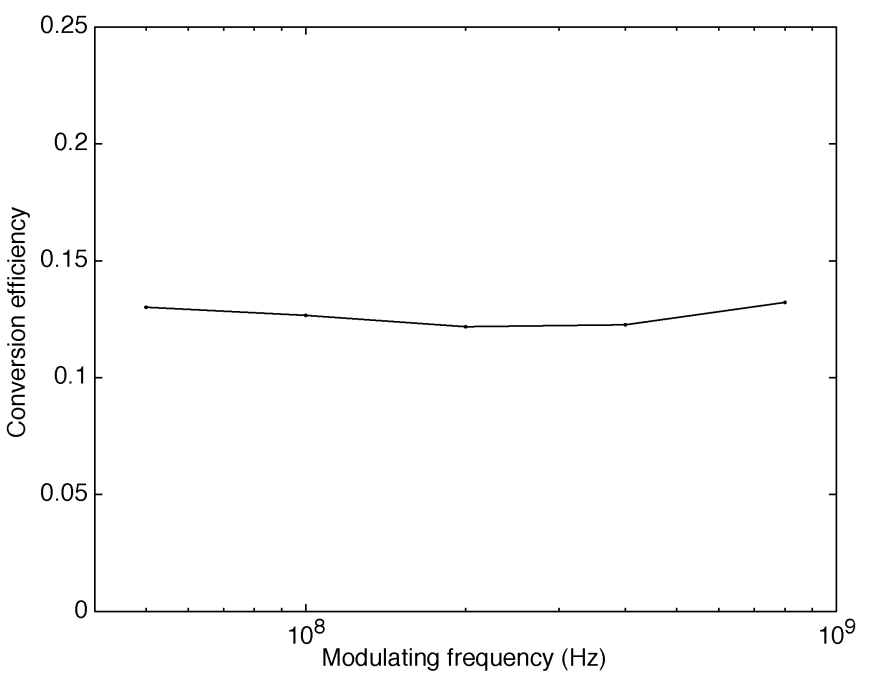

Fig. 4. Computed LFCE as a function of the modulating frequency between $50-800 \mathrm{MHz}$, maintaining $100 \%$ amplitude modulation of a $20-\mathrm{V} / \mathrm{cm} 4-\mathrm{GHz}$ microwave carrier. The conversion efficiency is approximately constant over the entire range of modulating frequencies.

$0.1 \mathrm{MHz}$, stimulation of excitable biological tissues plays a dominant role" [10], such a conversion phenomenon could have significant implications for human health and safety.

In conclusion, the results of our hydrodynamic model of the cellular sodium ion channel provide a formal nonlinear mechanism for transducing microwave signals to influence ion flow currents at time scales potentially permitting interaction with naturally occurring body processes. Such a nonthermal transduction would present possibilities for appropriately modulated microwave signals to interfere with normal cell-membrane functions. This could have potential impacts, especially in the defense technology area, where it is conceivable that personnel could be exposed to the required $2-\mathrm{kV} / \mathrm{m}$ microwave electric field. Finally, our computational modeling results may be useful in guiding experimental investigations of potential microwave nonlinearities in biological tissues [20].

\section{ACKNOWLEDGMENT}

The simulation code for the authors' model is adapted from that created by C.-W. Shu, Brown University, Providence, RI, and is used with permission.

\section{REFERENCES}

[1] M. Grandolfo, S. M. Michaelson, and A. Rindi, Eds., Biological Effects and Dosimetry of Nonionizing Radiation. New York: Plenum, 1983.

[2] R. Adey and A. F. Lawrence, Eds., Nonlinear Electrodynamics in Biological Systems. New York: Plenum, 1984.

[3] Biological Effects and Exposure Criteria for Radiofrequency Electromagnetic Fields. Bethesda, MD: Nat. Council Radiat. Protection Meas., 1986.

[4] C. Polk and E. Postow, Handbook of Biological Effects of Electromagnetic Fields, 2nd ed. Boca Raton, FL: CRC, 1995.

[5] B. J. Klauenberg, M. Grandolfo, and D. N. Erwin, Eds., Radiofrequency Radiation Standards: Biological Effects, Dosimetry, Epidemiology, and Public Health Policy. ser. NATO ASI, ser. A: Life Sciences. New York: Plenum, 1995, vol. 274.

[6] I. Rubinstein, Electro-Diffusion of Ions. Philadelphia, PA: SIAM, 1990. 
[7] C.-W. Shu and S. Osher, "Efficient implementation of essentially nonoscillatory shock-capturing scheme, II," J. Comput. Phys., vol. 83, pp. 32-78, 1989.

[8] E. Fatemi, J. Jerome, and S. Osher, "Solution of the hydrodynamic device model using high-order nonoscillatory shock-capturing algorithms," IEEE Trans. Computer-Aided Design, vol. 10, pp. 232-244, Feb. 1991.

[9] D.-P. Chen, R. S. Eisenberg, J. W. Jerome, and C.-W. Shu, "Hydrodynamic model of temperature change in the open ionic channel," Biophys. J., vol. 89, pp. 2304-2322, 1995.

[10] J. C. Lin, "ANSI/IEEE exposure standards for radiofrequency fields," in Radiofrequency Radiation Standards: Biological Effects, Dosimetry, Epidemiology, and Public Health Policy. ser. NATO ASI, ser. A: Life Sciences, B. J. Klauenberg, M. Grandolfo, and D. N. Erwin, Eds. New York: Plenum, 1995, vol. 274, pp. 31-33.

[11] B. Hille, Ionic Channels of Excitable Membranes, 2nd ed. Sunderland, MA: Sinauer Assoc., 1992.

[12] R. R. Llinás, "The intrinsic electrophysiological properties of mammalian neurons: Insights into central nervous system function," Science, vol. 242, pp. 1654-1664, Dec. 1988.

[13] H. Jahnsen and R. Llinás, "Ionic basis for the electroresponsiveness and oscillatory properties of guinea-pig thalmic neurones in vitro," $J$. Physiol. (London), vol. 349, pp. 227-247, 1984.

[14] J. Koester and S. A. Siegelbaum, "Membrane potential," in Principles of Neural Science, 4th ed, E. R. Kandel, J. H. Schwartz, and T. M. Jessel, Eds. New York: McGraw-Hill, 2000.

[15] B. Hille, C. M. Armstrong, and R. MacKinnon, "Ion channels: From idea to reality," Nature Med., vol. 5, pp. 1105-1109, Oct. 1999.

[16] S. A. Siegelbaum and J. Koester, "Ion channels," in Principles of Neural Science, 4th ed, E. R. Kandel, J. H. Schwartz, and T. M. Jessel, Eds. New York: McGraw-Hill, 2000.

[17] C. M. Armstrong and B. Hille, "Voltage-gated ion channels and electrical excitability," Neuron, vol. 20, pp. 371-380, Mar. 1998.

[18] D. A. Doyle, J. M. Cabral, R. A. Pfuetzner, A. Kuo, J. M. Gulbis, S. L. Cohen, B. T. Chait, and R. MacKinnon, "The structure of the potassium channel: Molecular basis of $K^{+}$conduction and selectivity," Science, vol. 280, pp. 69-77, Apr. 1998.

[19] G. Baccarani and M. Wordeman, "An investigation of steady-state velocity overshoot effects in Si and GaAs devices," Solid State Electron., vol. 28, pp. 407-416, 1985.

[20] Q. Balzano, "Proposed test for detection of nonlinear responses in biological preparations exposed to RF energy," Bioelectromagnetics, vol. 23, pp. 278-287, 2002.

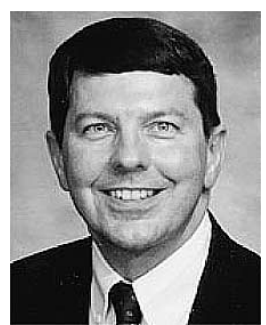

Joseph W. Jerome received the Ph.D. degree in mathematics from Purdue University, West Lafayette, IN, in 1966.

From 1966 to 1968, he was a Visiting Assistant Professor with the Mathematics Research Center, University of Wisconsin. From 1968 to 1970, he was an Assistant Professor with Case Western Reserve University. In 1970, he joined Northwestern University, Evanston, IL, where, since 1976, he has been a Professor of mathematics and applied mathematics. He has held sabbatical positions with Oxford University, Oxford, U.K. (1974-1975), The University of Texas at Austin (1978-1979), and Bell Laboratories, Murray Hill, NJ (1982-1983). In 1985, he was a Visiting Scholar with the University of Chicago. He authored Analysis of Charge Transport: A Mathematical Study of Semiconductor Devices (Berlin, Germany: Springer-Verlag, 1996). He edited Modeling and Computation for Applications in Mathematics, Science, and Engineering (New York: Oxford, 1998). His research interests include partial differential equations, applied analysis, approximation theory, computational electronics, and ion transport in biology.

Dr. Jerome received the Distinguished Alumnus Award from Purdue University's School of Science in 1996.

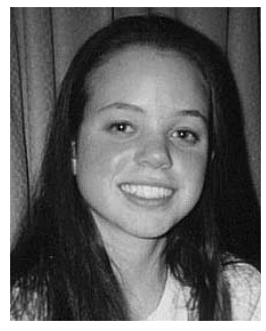

Lauren C. Pierce is a third-year student working toward the integrated seven-year combined B.S./M.D. degree at Northwestern University, Evanston, IL, as part of their Honors Program in Medical Education.

While at Northwestern University, she has been an Undergraduate Research Assistant with the Electromagnetics Laboratory, where she investigates the possibility of microwave electrical nonlinearities in cellular ion channels.

Ms. Pierce was the recipient of numerous academic honors.

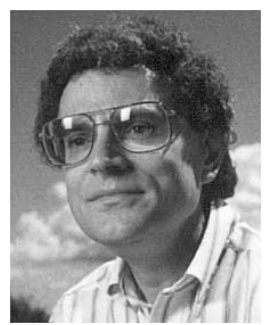

Allen Taflove (F'90) was born in Chicago, IL, on June 14, 1949. He received the B.S., M.S., and Ph.D. degrees in electrical engineering from Northwestern University, Evanston, IL, in 1971, 1972, and 1975, respectively.

After nine years as a Research Engineer with the IIT Research Institute, Chicago, IL, he returned to Northwestern University in 1984, where, since 1988, he has been a Professor in the Department of Electrical and Computer Engineering. Since 1972, he has pioneered basic theoretical approaches and engineering applications of finite-difference time-domain (FDTD) computational electrodynamics. He coined the FDTD acronym in a 1980 IEEE paper. FDTD is currently one of the most powerful and widely used methods for solving Maxwell's equations to model linear and nonlinear electromagnetic-wave interactions with electrically large and complex structures. He has been the thesis adviser of 18 Ph.D. recipients who hold professorial or technical staff positions at major institutions including research universities and national laboratories. He authored the popular textbook Computational Electrodynamics: The Finite-Difference Time-Domain Method (Boston, MA: Artech House, 1995; 2000, 2nd ed.). A third edition of this book is planned for 2005. In total, he has authored or coauthored four books, 12 book chapters, and approximately 90 journal papers and 200 conference papers. He holds 14 U.S. patents. These publications resulted in his being included on ISIHighlyCited.com, the Institute of Scientific Information's list of the most-cited researchers worldwide. His research interests span much of the electromagnetic spectrum. He and his students are currently modeling electrodynamic phenomena ranging from geophysically induced extremely low-frequency wave propagation about the entire Earth to novel emerging principles of optical ultramicroscopy. The principle that "Maxwell's equations work from dc to light" is vividly demonstrated in his laboratory every day.

Dr. Taflove currently serves as Master of Northwestern's 140-student Slivka Residential College of Science and Engineering, and as the faculty advisor to Northwestern's Undergraduate Design Competition, Undergraduate Research Journal, and the student chapters of Eta Kappa Nu and Tau Beta Pi. His efforts on behalf of students at all levels were recognized by Northwestern University in 2000 when he was named a C. D. McCormick Professor of Teaching Excellence. 